\title{
Necessidades de cuidados de enfermagem e intervenções terapêuticas em Unidade de Terapia Intensiva: estudo comparativo entre pacientes idosos e não idosos
}

\author{
Nursing care need and therapeutics interventions in Intensive Care Unit: \\ a comparative study among elderly and non-elderly patients \\ Necesidades de cuidados de enfermeria e intervenciones terapeuticas en la Unidad \\ de Cuidados Intensivos: estudio comparativo entre pacientes ancianos y no ancianos

\section{Juliana Trench Ciampone ${ }^{\mathbf{1}}$, Leilane Andrade Gonçalves ${ }^{\mathbf{1}}$, Flávia de Oliveira Motta Maia ${ }^{1}$, Kátia Grillo Padilha ${ }^{2}$}

\section{RESUMO}

Objetivos: comparar as necessidades de cuidados de enfermagem e as intervenções terapêuticas realizadas em pacientes idosos e não idosos em UTI, segundo o Nursing Activities Score (NAS). Métodos: Compuseram a amostra, 50 pacientes adultos admitidos na UTI de um Hospital Universitário do município de São Paulo, a partir de 26 de setembro de 2003. O teste de Mann-Whitney foi utilizado para a comparação entre as variáveis. Resultados: Da amostra de pacientes, a média de idade foi de 70 anos. O mais freqüente tipo de tratamento foi clínico (78,0\%), observando-se uma média de permanência de 3,5 dias e mortalidade de 38,0\%. A pontuação média NAS foi de $66,57 \%$ $( \pm 9,15)$ permanecendo acima de $60,0 \%$ durante o período analisado. Não se observou diferença na média NAS de pacientes idosos $(66,44 \%)$ e não idosos $(66,33 \%)$, assim como não houve diferença entre as intervenções terapêuticas realizadas nos dois grupos de pacientes. Conclusão: Os resultados mostram a necessidade de discussões sobre a indicação de UTI, frente às implicações éticas, econômicas e sociais inerentes à assistência intensiva.

Descritores: Cuidados de enfermagem; Terapêutica; Unidades de terapia intensiva; Cuidados intensivos; Idoso; Determinação de necessidades de cuidados de saúde.

\begin{abstract}
Objectives: To compare nursing care needs and therapeutic interventions of elderly and non-elderly patients admitted to an ICU. Methods: The sample consisted of 50 adult patients from a university hospital in the district of São Paulo. Data were collect with the Nursing Activity Scale (NAS) from September 26, 2003. The NAS was applied daily, from the time of admission to and discharge from the ICU, with a total of 339 measurements. Patients with 60 years of age or older were considered elderly patients. Data were analyzed with descriptive statistics and Mann-Whitney tests. Results: The mean age of the patient sample was 70 years and the most frequent type of treatment was clinical (78.0\%). The mean stay was 3.5 days and the mortality rate was $38.0 \%$. The mean score for NAS was $66.57 \%(+9.15)$ and remained above the $60.0 \%$ mark for the entire period analyzed. No difference was observed between the mean NAS scores for elderly (66.44\%) and nonelderly $(66.33 \%)$ patients. And, there was no difference between the therapeutic interventions carried out in the two groups of patients. Conclusions: The results show the need for further discussion about the criteria for admission in ICU regarding the ethical, social, and economic implications of intensive care.
\end{abstract}

Keywords: Nursing care; Therapeutic; Intensive care unit; Intensive care; Aged; Needs assessment.

\begin{abstract}
RESUMEN
Objetivos: comparar las necesidades de cuidados de enfermería y las intervenciones terapéuticas realizadas en pacientes adultos mayores y no adultos mayores en la UCI, según el Nursing Activities Score (NAS). Método: Hicieron parte de la muestra, 50 pacientes adultos admitidos en la UCI de un Hospital Universitario del municipio de Sao Paulo, a partir del 26 de setiembre del 2003. El NAS fue aplicado diariamente totalizando 339 medidas. Fueron considerados como adultos mayores los pacientes con edad igual o superior a 60 años. El test de Mann-Whitney fue utilizado para la comparación entre las variables. Resultados: De la muestra de pacientes, el promedio de edad fue de 70 años. El tipo más frecuente de tratamiento fue el clínico $(78,0 \%)$. Se observó un promedio de permanencia de 3,5 días y una mortalidad de 38,0\%. La puntuación promedio NAS fue de 66,57\% (+9,15) manteniéndose sobre el 60,0\% durante el período analizado. No se observó diferencia en el promedio NAS de pacientes adultos mayores $(66,44 \%)$ y no adultos mayores $(66,33 \%)$. De esta forma, no hubo diferencia entre las intervenciones terapéuticas realizadas en los dos grupos de pacientes. Conclusion: Los resultados muestran la necesidad de discusiones sobre la indicación de la UCI, frente a las implicaciones éticas, económicas y sociales inherentes a la atención intensiva. Descriptores: Atención de enfermería; Terapêutica; Unidades de terapia intensiva; Cuidados intensivos; Anciano; Evaluación de
\end{abstract} necesidades.

${ }^{1}$ Enfermeira da Unidade de Terapia intensiva do Hospital Universitário da Universidade de São Paulo (SP), Brasil.
${ }^{2}$ Professor Associado do Departamento Enfermagem Médico-Cirúrgica da Escola de Enfermagem da Universidade de São Paulo (SP), Brasil. 


\section{INTRODUÇÃO}

As Unidades de Terapia Intensiva (UTIs) são unidades complexas, destinadas ao atendimento de pacientes graves, que demandam espaço físico específico, recursos humanos especializados e instrumental tecnológico avançado, o que as tornam unidades de alto custo. Dessa forma, torna-se necessária a definição de critérios de internação e alta de pacientes na UTI que considere os diversos aspectos envolvidos na indicação do tratamento intensivo, com vistas a beneficiar o paciente e otimizar recursos $^{(1)}$.

Nesse sentido, a idade é um dos fatores a serem considerados, uma vez que traz questionamentos quanto à aplicação de recursos na UTI e a relação custo-benefício nessas unidades. A população está envelhecendo, e com isso o número de pessoas com doenças crônicodegenerativas vêm aumentando ${ }^{(2)}$, levando a agravos à saúde com conseqüente necessidade de intervenções que requerem internação na UTI.

No Brasil, o rápido e contínuo aumento do número de idosos (e $>60$ anos de idade) que passou de 3 milhões em 1960, para 7 milhões em 1975 e 14 milhões em 2002, com aumento de $500 \%$ em quarenta anos, e estimativa que alcançará 32 milhões em 2020(2), tem provocado discussões relacionadas ao atendimento à saúde, com repercussões nas UTIs. Isto porque, nesse cenário, também os custos do tratamento intensivo vêm crescendo continuamente com a possibilidade de que, no futuro, o acesso à terapia intensiva venha a ser restrita aos idosos ${ }^{(1)}$.

Um dilema em relação ao tratamento de pacientes idosos na UTI consiste em decidir em que medida investimentos terapêuticos devem ser aplicados diante de perspectivas reduzidas de sobrevida ou de limitada qualidade de vida após a alta da Unidade, sem transgredir princípio ético de igualdade de atendimento, assegurado pelo estatuto do idoso ${ }^{(3)}$, em que a idade não pode ser fator de exclusão.

A literatura internacional é controversa no que diz respeito à idade como elemento preditor isolado para o prognóstico em UTI e, conseqüentemente, para a aplicação de recursos disponíveis nessas Unidades. De acordo com Rocker $^{(4)}$, fatores tais como o estado funcional do paciente previamente à internação na UTI, a necessidade de ventilação mecânica, a presença de doenças malignas e o número de disfunções orgânicas também são importantes como elementos prognósticos. Entretanto, segundo afirmação de Barreto e colaboradores $^{(1)}$ estudos demonstram que a sobrevida em um ano após a alta de uma UTI é cerca de 56,0\% para pacientes com idades entre $70-85$ anos e de $27,0 \%$ para pacientes com mais de 85 anos. Também, em alguns casos, a análise multivariada identificou a idade como fator independente para a mortalidade.
A despeito de o idoso apresentar altas taxas de reinternação e óbito no período de 1 ano, de ser remota a possibilidade de recuperação da sua capacidade funcional anterior a internação hospitalar ${ }^{(5)}$ e de estudos demonstrarem alta mortalidade na $\mathrm{UTI}^{(6)}$, a assistência intensiva, indicada com base nos recursos hoje disponíveis, deve assegurar ao idoso o melhor tratamento médico e de enfermagem disponíveis.

É nesse contexto que surge o interesse deste estudo em saber se, uma vez na UTI, os investi-mentos terapêuticos variam em relação à idade dos pacientes. Questiona-se, além disso, se as necessidades de cuidados de enfermagem, considerando-se as intervenções terapêuticas, diferem entre pacientes idosos e não idosos.

Com a disponibilidade de instrumentos de medida como o Nursing Activities Score (NAS) ${ }^{(7)}$ que avaliam objetivamente a necessidade de cuidados que os pacientes requerem, tendo por base a quantificação das intervenções realizadas, tornou-se possível buscar respostas a algumas dessas questões.

O NAS tem em sua composição 7 categorias de intervenções terapêuticas que são subdivididas formando um conjunto de 23 itens. Para cada item há uma pontuação, que quando somadas formam o escore total NAS, o qual expressa a porcentagem de tempo gasto por um profissional de enfermagem na assistência direta ao doente crítico, em um turno de trabalho na $\mathrm{UTI}^{(7)}$. Assim, considerando a idade como um fator importante a ser investigado na UTI, pretendeu-se com este estudo obter informações sobre as necessidades de cuidados e as intervenções terapêuticas realizadas em pacientes idosos na UTI, como subsídios para a discussão das implicações da assistência intensiva a esse grupo etário.

\section{OBJETIVOS}

- Comparar as necessidades de cuidados de enfermagem de pacientes idosos e não idosos em UTI segundo o NAS.

- Comparar as categorias de intervenções terapêuticas do NAS realizadas em pacientes idosos e não idosos internados na UTI.

- Comparar as intervenções terapêuticas realizadas em pacientes idosos e não idosos internados na UTI, segundo o NAS.

\section{MÉTODOS}

O estudo, de abordagem quantitativa, foi desenvolvido na Unidade de Terapia Intensiva (UTI) de adultos, do Hospital Universitário da Universidade de São Paulo, localizado no município de São Paulo, instituição de atendimento secundário, que dispõe de um total de 11 leitos de UTI e igual número destinado à Unidade Semi- 
Intensiva (U.S.I.).

A população foi constituída por 50 pacientes com idade igual ou superior a 18 anos, admitidos consecutivamente, na UTI, a partir de 26 de setembro de 2002 , e que nela permaneceram por um período mínimo de 24 horas. Readmissões foram excluídas do estudo.

Para a coleta de dados utilizou-se um impresso contendo dados sócio-demográficos e clínicos, além do NAS, com a discriminação dos seus itens componentes (ver ANEXO).

Após a aprovação do projeto pela Comissão de Ética em Pesquisa do hospital campo de estudo (protocolo $\mathrm{n}^{\circ}$ 294/02), foi feito o acompanhamento prospectivo dos pacientes, da internação à alta da UTI, até a saída do último paciente da amostra, o que ocorreu no dia 03 de novembro de 2002. Atendido o critério de inclusão e com o Termo de Consentimento Livre e Esclarecido assinado pelo paciente ou seu responsável legal, procedeu-se o preenchimento do NAS, tendo por base dados do prontuário e ficha de controle dos pacientes, acrescidos de informações complementares fornecidas pelos enfermeiros, sempre que necessário. Para fins de padronização, foram consideradas as informações referentes às 24 horas do dia anterior que se completavam às 8 horas da manhã. Quanto ao primeiro dia de internação, foram computadas as intervenções realizadas da hora da admissão na UTI até às 8 horas do dia seguinte, independente de completarem ou não 24 horas. Também no dia da alta, além dos dados registrados às 8 horas da manhã, foram computados aqueles relacionados ao período compreendido entre 8 horas e o momento de saída.

Quanto às faixas etárias, os pacientes foram divididos em dois grupos, ou seja, indivíduos adultos com idade inferior a 60 anos e idosos, com idade igual ou superior a 60 anos, segundo preconizado pelo Estatuto do Idoso(7).

Os dados foram armazenados em um banco eletrônico criado no Microsoft ${ }^{\circledR}$ Excel 2000 e as análises estatísticas realizadas através do SPSS ${ }^{\circledR} 10.0$ for Windows (Statistical Package For The Social Sciences). Os resultados referentes às características demográficas, necessidades de cuidados de enfermagem e intervenções terapêuticas foram submetidos à análise estatística.

Para o estudo comparativo das necessidades de cuidados de enfermagem com a variável idade, foi aplicado o teste não paramétrico de Mann-Whitney, também empregado para a comparação das categorias e itens do NAS com a faixa etária dos pacientes.

Foram considerados estatisticamente significantes os resultados cujos valores de $p$ foram inferiores a 0,05 .

\section{RESULTADOS}

\footnotetext{
4.1. Caracterização dos dados demográficos, das necessidades de cuidados e das intervenções terapêuticas na amostra de pacientes.
}

Do total de 50 pacientes que compuseram o estudo, foram obtidas 339 medidas do escore NAS.

Em relação às características demográficas da amostra, houve igual distribuição de pacientes do sexo masculino e feminino, ambos com 50,0\%. A média de idade foi de 70 anos (+15,9), com variação entre 20 e 91 anos. Pacientes com idade igual ou acima de 60 anos foram a maioria $(66,0 \%)$, seguidos de $34,0 \%$ com idade entre 21 e 60 anos. Verificou-se que $84,0 \%$ eram portadores de doença crônica pré-existente, dos quais $61,9 \%, 35,7 \%$ e $2,4 \%$ apresentaram, respectivamente, 1,2 e 3 doenças crônicas. Internações por motivos clínicos $(78,0 \%)$ e doenças do sistema cardiovascular $(70,0 \%)$ foram predominantes.

Quanto aos dados da internação, $40,0 \%$ dos pacientes foram provenientes do Pronto Socorro (PS), 24,0\% da Unidade Semi-Intensiva (U.S.I.) e $20,0 \%$ do Centro Cirúrgico (CC), sendo que a maioria $(78,0 \%)$ apresentou problema clínico como motivo da internação na UTI. Dos $22,0 \%$ submetidos à cirurgia, apenas $2(9,1 \%)$ foram submetidos à cirurgia eletiva. A média de permanência foi de 3,5 dias, com variação de 1 a 26 dias, verificando-se que $64,0 \%$ dos pacientes permaneceram na UTI por um período de 1 a 5 dias. Igual porcentagem (18,0\%) estiveram internados de 6 a 10 e mais do que 10 dias. Referente ao destino após a saída da UTI, $54,0 \%$ dos pacientes foram transferidos para a U.S.I. e 38,0\% foram a óbito.

A análise das necessidades de cuidados e, conseqüentemente, de demanda de trabalho de enfermagem mostrou uma média NAS de $66,5 \%(+9,1)$, mediana de $70,7 \%$, com variação de $47,6 \%$ a $82,4 \%$.

Referente às intervenções terapêuticas realizadas durante a internação na UTI, verificou-se que 100,0\% dos pacientes foram submetidos às seguintes intervenções: presença à beira do leito e observação contínua ou ativa por 4 horas ou mais em algum plantão; investigações laboratoriais; medicação e procedimentos de higiene de rotina. Também número elevado de pacientes teveram como intervenções: a realização de tarefas administrativas e gerenciais de rotina que exigiram menos de duas horas (99,7\%); suporte respiratório; tratamento para melhora da função pulmonar; suporte e cuidados aos familiares e pacientes por cerca de 1 hora (99,4\%, cada um); seguidos da medida quantitativa do débito urinário $(88,8 \%)$ e cuidados com drenos $(87,3 \%)$.

Além das intervenções mencionadas, $71,7 \%$ dos pacientes necessitaram de cuidados com as vias aéreas artificiais, 64,3\% de mobilização e posicionamento mais do que 3 vezes em 24 horas; 56,9\% de alimentação enteral e 54,0\% de medicação vasoativa, independente do tipo e dose.

As demais intervenções contidas no NAS foram realizadas em menos de $20,0 \%$ dos pacientes, observando- 
Tabela 1. Média e desvio-padrão das intervenções terapêuticas em UTI segundo faixa etária. São Paulo, 2003.

\begin{tabular}{|c|c|c|c|c|}
\hline \multirow{2}{*}{ Intervenções Terapêuticas } & & \multicolumn{2}{|c|}{ Idade } & \multirow{2}{*}{ p-valor' } \\
\hline & & $<60$ anos $(n=17)$ & $\geq 60$ anos $(n=33)$ & \\
\hline Monitorização e controles & Média (DP) & $19,60(0,00)$ & $19,60(0,00)$ & $* * *$ \\
\hline Investigações Laboratoriais & Média (DP) & $4,30(0,00)$ & $4,30(0,00)$ & $* * *$ \\
\hline Medicação, exceto droga vasoativa & Média (DP) & $5,60(0,00)$ & $5,60(0,00)$ & $* * *$ \\
\hline Procedimentos de Higiene & Média (DP) & $4,10(0,00)$ & $4,10(0,00)$ & $* * *$ \\
\hline Cuidados com drenos & Média (DP) & $1,62(0,46)$ & $1,60(0,53)$ & 0,763 \\
\hline Mobilização e Posicionamento & Média (DP) & $7,27(5,82)$ & $7,37(5,43)$ & 0,810 \\
\hline Suporte e cuidados aos familiares e pacientes & Média (DP) & $4,18(0,78)$ & $4,00(0,00)$ & 0,182 \\
\hline Tarefas administrativas e gerenciais & Média (DP) & $4,26(0,26)$ & $4,20(0,00)$ & 0,182 \\
\hline Suporte respiratório & Média (DP) & $1,40(0,00)$ & $1,36(0,25)$ & 0,453 \\
\hline Cuidado com vias aéreas artificiais & Média (DP) & $0,88(0,79)$ & $0,99(0,83)$ & 0,624 \\
\hline Tratamento para melhora da função pulmonar & Média (DP) & $4,40(0,00)$ & $4,26(0,78)$ & 0,453 \\
\hline Suporte cardiovascular & Média (DP) & $0,49(0,51)$ & $0,58(0,56)$ & 0,448 \\
\hline Reposição de grandes perdas de fluídos & Média (DP) & $0,45(0,64)$ & $0,26(0,48)$ & 0,121 \\
\hline Monitorização do átrio esquerdo & Média (DP) & $0,16(0,35)$ & $0,03(0,12)$ & 0,086 \\
\hline Reanimação cardiorrespiratória & Média (DP) & $0,15(0,47)$ & $0,04(0,16)$ & 0,492 \\
\hline Suporte renal & Média (DP) & $0,00(0,00)$ & $0,39(1,40)$ & 0,185 \\
\hline Medida quantitativa do débito urinário & Média (DP) & $6,31(1,81)$ & $6,38(1,97)$ & 0,508 \\
\hline Suporte neurológico & Média (DP) & $0,00(0,00)$ & $0,00(0,00)$ & 1,000 \\
\hline Suporte metabólico & Média (DP) & $0,04(0,15)$ & $0,08(0,20)$ & 0,221 \\
\hline Hiperalimentação intravenosa & Média (DP) & $0,29(0,72)$ & $0,10(0,42)$ & 0,244 \\
\hline Alimentação enteral & Média (DP) & $0,45(0,59)$ & $0,68(0,61)$ & 0,121 \\
\hline Intervenções específicas na UTI & Média (DP) & $0,24(0,34)$ & $0,27(0,56)$ & 0,722 \\
\hline Intervenções específicas fora da UTI & Média (DP) & $0,13(0,25)$ & $0,25(0,42)$ & 0,231 \\
\hline
\end{tabular}

se que, à exceção dos itens mutuamente excludentes, nenhum recebeu pontuação no item suporte neurológico.

4.2. Necessidades de cuidados de enfermagem e intervenções terapêuticas segundo a faixa etária

Buscando-se investigar a influência da idade nas necessidades de cuidados de enfermagem, a análise dos dados mostrou que não houve diferença estatisticamente significativa entre os grupos analisados, encontrando-se pontuação média diária NAS de $66,4 \%(+9,17)$ nos pacientes idosos e de 66,3\% (+9,41) nos não idosos $(\mathrm{p}=0,968)$.

Também as categorias de intervenções terapêuticas, segundo a faixa etária, não foram diferentes entre os dois grupos. Pacientes idosos e não idosos foram submetidos ao mesmo conjunto de intervenções, ou seja, atividades básicas $(\mathrm{p}=0,992)$, suporte ventilatório $(\mathrm{p}=0,671)$, suporte cardiovascular $(p=0,764)$, suporte renal $(p=0,216)$, suporte metabólico $(\mathrm{p}=0,519)$ e intervenções específicas $(\mathrm{p}=0,480)$.

Conforme dados da Tabela 1, também não houve diferença entre os grupos quando se analisou cada uma das intervenções realizadas na UTI.

\section{DISCUSSÃO}

Quanto à variável idade, os resultados encontrados são semelhantes aos achados de estudos nacionais e internacionais, apontando um número elevado de pacientes idosos em UTIs ${ }^{(7-10)}$. Observou-se que dos 33 pacientes com idade igual e maior de 60 anos, 16 (50,0\%) tinham entre 71 e 80 anos, 8 (25,0\%), entre 81 e 90 anos e um paciente $(3,1 \%), 91$ anos, reiterando a presença de pacientes com idade avançada submetidos a tratamento intensivo na UTI. Quanto ao gênero, os resultados confirmam a literatura, pois, apesar dos estudos apontarem predominância do sexo masculino nas UTIs, observa-se uma distribuição bastante próxima do sexo feminino $^{(10-11)}$.

Quanto à existência de doenças crônicas prévias, a constatação de que a maioria dos doentes $(84,0 \%)$ tinha, em média, 1,4 doenças, com predomínio de alterações do sistema cardiovascular, encontra semelhança em estudo realizado em UTIs do município de São Paulo, onde $70,9 \%$ dos pacientes apresentavam algum tipo de antecedente clínico relacionado à doenças do aparelho circulatório $^{(11)}$, o que era esperado em razão de serem as alterações do sistema circulatório, a primeira causa de mortalidade entre os idosos ${ }^{(12)}$.

Sobre a procedência dos pacientes para a UTI, estudos internacionais e nacionais ${ }^{(7,9,11)}$ apontam que a maior parte dos pacientes é proveniente do Centro Cirúrgico (C.C), seguido dos Serviços de Urgência (PS), diferindo, 
portanto, dos resultados desta investigação, que apresentou $40,0 \%$ dos pacientes vindos do PS, seguidos de $24,0 \%$ provenientes da U.S.I. No presente estudo, considerando que a maioria dos pacientes eram idosos, que grande parte apresentava doenças crônicas pré-existentes e que também a maioria das internações ocorreu por razões clínicas, pode-se inferir que o conjunto dessas variáveis tenha levado os doentes já instáveis internados no PS e na U.S.I. a serem atendidos na UTI.

No que se refere ao tempo de permanência predominante, de 1 a 5 dias, os resultados são corroborados pelo Censo Brasileiro de UTIs, realizado pela Associação de Medicina Intensiva Brasileira ${ }^{(13)}$, de 1 a 6 dias, em conformidade com as UTIs do país.

A alta mortalidade encontrada, ou seja, 38,0\%, situase não só acima da verificada em estudos brasileiros, com variação de $29,0 \%$ a $35,0 \%{ }^{(10,14)}$, como também internacionais, que oscilaram entre 8,0 e $19,0 \% 0^{(7,15)}$. Observa-se, além disso, que a mortalidade neste estudo foi maior, inclusive do que a encontrada em estudo realizado nesta mesma unidade um ano antes, que foi de $33,0 \%{ }^{(16)}$. A constatação da elevada mortalidade no período analisado, talvez se justifique pela associação de diferentes fatores, destacando-se, além da idade avançada, a existência de estados mórbidos pré-existentes, a doença atual e a gravidade clínica, não se podendo descartar as admissões na UTI de pacientes fora de possibilidades terapêuticas.

Ao se investigar os escores NAS, cuja média foi de $66,5 \%(+9,15)$, mediana de $70,7 \%$, portanto elevados quando se consideram as necessidades de cuidados e, conseqüentemente, a carga de trabalho de enfermagem na UTI, os resultados mostram dados importantes. Considerando-se que o NAS total representa o tempo gasto por um profissional de enfermagem para o cuidado de um paciente, em um plantão, as pontuações que atingem cerca de $70,0 \%$, demandam uma relação funcionário/paciente maior do que 1:2 preconizada pelas Normas e critérios para a criação de UTIs, do Ministério da Saúde ${ }^{(17)}$. Portanto, este aspecto deve ser levado em conta pelos enfermeiros para o dimensionamento adequado de pessoal e garantia da qualidade da assistência.

Sobre as intervenções terapêuticas a que os pacientes foram submetidos, os resultados mostraram semelhanças aos encontrados em uma amostra de 200 pacientes de um hospital particular do município de São Paulo utilizada na validação do $\mathrm{NAS}^{(8)}$. Notou-se, porém, que cuidados com drenos; realização de procedimentos de mobilização e posicionamento mais do que 3 vezes em 24 horas; uso de medicação vasoativa; alimentação enteral e cuidado com vias aéreas artificiais foram mais freqüentes nesta investigação.

Também estudo realizado na Alemanha, com o TISS28 , instrumento que precedeu o NAS, reitera os resultados obtidos, quando se analisa a prevalência das intervenções terapêuticas comuns nos dois instrumentos. Apenas duas exceções foram verificadas e incluíram a utilização de drogas vasoativas e alimentação enteral, ambos com freqüências menores do que as encontradas neste estudo ${ }^{(15)}$.

A análise comparativa dos resultados obtidos no presente estudo com as referências mencionadas, leva a supor que os investimentos terapêuticos foram maiores na amostra deste estudo, apontando possivelmente para uma maior gravidade dos pacientes que exigiram elevado número de intervenções para o tratamento das disfunções orgânicas existentes. Da mesma forma, parece justificada a expressiva necessidade de cuidados de enfermagem mensurada pelo NAS, de cerca de 70,0\% do tempo de trabalho de um profissional de enfermagem, observada também quando se analisou, comparativamente, pacientes idosos e não idosos.

Quanto ao volume de intervenções realizadas, foi interessante constatar que não houve diferença na aplicação dos recursos terapêuticos entre pacientes idosos e não idosos, demonstrando que, uma vez admitidos na UTI, independente da idade, os pacientes receberam todos os investimentos disponíveis para o seu tratamento.

No entanto, mais importante do que analisar se as intervenções realizadas nos pacientes idosos diferem dos não idosos depois de admitidos na UTI, os resultados levam a pensar na indicação de internação na UTI, em razão do elevado custo financeiro, físico e emocional inerente ao atendimento nessa Unidade. Obviamente, não se trata de desestimular a internação de idosos na UTI, mesmo porque, na realidade nacional, maior número de estudos devem ser desenvolvidos no sentido de analisar as condições de saída do paciente da Unidade e do hospital. Trata-se, porém, de aprofundar a discussão sobre a questão com vistas ao estabelecimento de diretrizes que contribuam para essa indicação. Isso se justifica, em razão dos aspectos éticos cruciais que podem se apresentar, após a admissão na UTI, e que incluem quando interromper os investimentos frente aos princípios da beneficência, não maleficência, justiça e qualidade de vida, complexos quando se trata de pacientes idosos ${ }^{(18,19)}$.

A constatação neste estudo que as necessidades de cuidados, as categorias de intervenções e as intervenções terapêuticas individualmente não foram diferentes entre pacientes idosos e não idosos demonstram que, uma vez admitidos na UTI, a equipe multiprofissional não mede esforços no sentido de recuperar as condições clínicas dos doentes. Porém, ainda que tais condutas sejam edificantes, deve-se ter em conta que embora os avanços científicos e tecnológicos tenham ampliado a expectativa de vida, a morte continua existindo, e quando ocorre não deve ser vista como fracasso da equipe ${ }^{(20-21)}$.

Outro aspecto a ser ressaltado no que se refere aos investimentos terapêuticos, consiste em levar em conta 
os desejos e expectativas do paciente e família com vistas a um resultado que possibilite a melhor qualidade de vida possível após a alta da unidade.

Nesse sentido, segundo Rocker ${ }^{(4)}$ estudo americano revelou que, ao serem questionados sobre seu tratamento, $60,0 \%$ dos pacientes responderam que gostariam de ter a dor e o desconforto aliviados, mesmo que isso significasse não viver por muito tempo. Os autores constataram, porém, que a opção pelo tratamento variou conforme a faixa etária. Enquanto $61,0 \%$ dos doentes com menos de 50 anos preferiam prolongar a vida a qualquer custo, essa proporção caiu para 52,0\%, 44,0\%, $37,0 \%$ e $27,0 \%$, respectivamente, nas faixas etárias de 50 a 59 anos, 60 a 69, 70 a 79 e mais de 80 anos.

Finalizando, pode-se dizer que enfrentar realisticamente os limites impostos pela condição humana de mortalidade constitui o grande desafio dos profissionais de saúde, uma vez que os problemas a serem enfrentados serão cada vez mais de natureza ética do que técnica, reafirmando a necessidade de atenção voltada à pessoa que está doente e não à sua doença ${ }^{(21,22)}$.

Apesar das limitações do estudo, decorrentes de ter sido realizado em uma única Unidade, de contar com uma amostra pequena e não associar a idade com outras variáveis, como a gravidade dos pacientes, os dados possibilitam reflexões a respeito da assistência intensiva a pacientes idosos, pois a magnitude do problema traz inerentes implicações que afetam paciente, família, instituição e aplicação de recursos na área da saúde.

\section{CONCLUSÔES}

Os resultados desta investigação permitiram as seguintes conclusões:

- pacientes idosos e não idosos internados na UTI, apresentaram iguais necessidades de cuidados e, conseqüen-temente, demanda de trabalho de enfermagem, com pontuação média de NAS de 66,44\% e 66,33\%, respectivamente;

- não houve diferença entre o grupo de idosos e não idosos quanto às categorias do NAS atividades básicas, suporte ventilatório, suporte cardiovascular, suporte renal, suporte metabólico e intervenções específicas;

- durante a internação na UTI, não houve diferença entre as intervenções terapêuticas recebidas pelo grupo de pacientes idosos e não idosos.

\section{REFERÊNCIAS}

1. Barreto SM, Vieira SRR, Pinheiro CTS. Rotinas em terapia intensiva. 3.ed. Porto Alegre: Artes Médicas; 2001.

2. Fundação IBGE. Censo demográfico 2000 [texto na Internet].[citado 2004 Dez 27]. Disponível em: $<$ :http://www.ibge.gov.br
3. Brasil. Lei 10741, de $1^{\circ}$ de outubro de 2003. Estatuto do idoso. Diário Oficial da União, Brasília, 02 out. 2003.

4. Rocker G. Controversial issues in critical care for the elderly: a perspective from Canada. Crit Care Clin. 2003; 19(4): 811-25.

5. Kalache A, Veras RP, Ramos LR. O envelhecimento da população mundial: um desafio novo. Rev Saúde Pública. 1987; 21(3): 200-10.

6. Nagappan R, Parkin G. Geriatric critical care. Crit Care Clin. 2003; 19(2): 253-70. Review.

7. Miranda DR, Nap R, de Rijk A, Schaufeli W, Iapichino G; TISS Working Group. Therapeutic Intervention Scoring. Nursing activities score. Crit Care Med. 2003; 31(2): 374-82.

8. Queijo AF. Tradução para o português e validação de um instrumento de medida de carga de trabalho de enfermagem em unidade de terapia intensiva: Nursing Activities Score (NAS). [Tese Mestrado]. São Paulo: Escola de Enfermagem da Universidade de São Paulo; 2002.

9. Pierin AMG, Padilha KG, Cruz DALM. Caracterização dos pacientes de duas unidades de Terapia Intensiva (UTI): condições bio-sociais, processo de internação e intervenções terapêuticas. Rev Esc Enferm. USP. 1990; 24(3): 371-88.

10. Paiva SAR, Matai O, Resende NO, Campana AO. Análise de uma população de doentes atendidos em unidade de terapia intensiva - estudo observacional de sete anos (1992-1999). Rev Bras Ter Intensiva. 2002; 14(2):73-80.

11. Silva MCM, Sousa RMC. Caracterização dos pacientes adultos e adolescentes das unidades de terapia intensiva do município de São Paulo. Rev Paul Enferm. 2002; 21(1):50-9.

12. Ministério da Saúde (BR). DATASUS. Indicadores e dados básicos - Brasil - 2003 (IDB - 2003) [texto na Internet]. [citado 2004 Dez 17]. Disponível em: http://tabnet.datasus.gov.br/cgi/idb2003/ folder.htm

13. Associação de Medicina Intensiva Brasileira - AMIB. $1^{\circ}$ Anuário Brasileiro de Terapia Intensiva; 1999/2000.

14. Livianu J, Anção MS, Andrei AM, Faria, LS. Índices prognósticos em unidade de terapia intensiva. In: Knobel E. Condutas no paciente grave. São Paulo: Atheneu; 1994. cap. 67, p. 823-33.

15. Lefering R, Zart M, Neugebauer EA. Retrospective evaluation of the simplified Therapeutic Intervention Scoring System (TISS-28) in a surgical intensive care unit. Intensive Care Med. 2000; 26(12): 1794-802.

16. Ducci AJ, Padilha KG, Telles SCR, Gutierrez BAO. Gravidade de pacientes e demanda de trabalho de enfermagem em Unidade de Terapia Intensiva: Análise evolutiva segundo o TISS-28. Rev Bras Ter 
Intensiva. 2004; 16(1): 22-7.

17. Brasil. Ministério da Saúde. Portaria no 3432 de 12 de agosto de 1998. Estabelece critérios de Classificação para as Unidades de Tratamento Intensivo-UTI [texto na Internet]. [citado 2005 Jan 31]. Disponível em: http// www.amib.com.br

18. Moritz RD. Dilemas éticos sobre o fim da vida. Rev Bras Ter Intensiva. 2003; 15(1):3-4.

19. Azevêdo ES. Bioética e medicina intensiva. Rev Bras Ter Intensiva. 1996; 8(4): 174-8.
20. Esslinger I. O paciente, a equipe de saúde e o cuidador: de quem é a vida, afinal?...um estudo acerca do morrer com dignidade [tese]. São Paulo: Instituto de Psicologia da Universidade de São Paulo; 2003.

21. Mularski RA, Osborne ML. End-of-life care in the critically ill geriatric population. Crit Care Clin. 2003; 19(4):789-810; viii.

22. Piva JP, Carvalho PRA. Considerações éticas nos cuidados médicos do paciente terminal. Bioética.1993; 1(2): 129-38.

\section{ANEXO}

Nome:

Idade:

Sexo:

Data da internação:

Procedência: ( )CM ( ) Centro-Cirúrgico ( )PS ( )Semi-Intensiva ( ) Clínica Cirúrgica ( )Outro Tipo de internação: ( )médica ( ) cirúrgica-eletiva ( )cirúrgica-urgência Diagnóstico: Doença crônica: ( )Hepática ( )Cardiovascular ( )Respiratória ( )Renal ( )Imunocomprometimento Data de saída: _______ Tipo de saída: ( )UI ( )óbito ( )semi-intensiva ( )transferência - outro hospital

\begin{tabular}{|c|c|}
\hline NAS & Pont. \\
\hline \multicolumn{2}{|l|}{ 1. Monitorização e Controles } \\
\hline 1a. Sinais vitais horário, cálculo e registro do balanço hídrico. & 4,5 \\
\hline 1b. Presença à beira do leito e observação ou atividade contínua por 2 horas ou mais em algum plantão. & 12,1 \\
\hline 1c. Presença à beira do leito e observação ou atividade contínua por 4 horas ou mais em algum plantão. & 19,6 \\
\hline 2. Investigações Laboratoriais: bioquímicas e microbiológicas. & 4,3 \\
\hline 3. Medicação, exceto drogas vasoativas. & 5,6 \\
\hline \multicolumn{2}{|l|}{ 4. Procedimentos de higiene } \\
\hline 4a. Realização de procedimentos de higiene. & 4,1 \\
\hline 4b. Realização de procedimentos de higiene que durem mais do que 2 horas, em algum plantão. & 16,5 \\
\hline 4c. Realização de procedimentos de higiene que durem mais do que 4 horas em algum plantão. & 20,0 \\
\hline 5. Cuidados com drenos: Todos (exceto sonda gástrica). & 1,8 \\
\hline \multicolumn{2}{|l|}{ 6. Mobilização e posicionamento } \\
\hline 6a. Realização do(s) procedimento(s) até 3 vezes em 24 horas. & 5,5 \\
\hline $\begin{array}{l}\text { 6b. Realização do(s) procedimento(s) mais do que } 3 \text { vezes em } 24 \text { horas ou com } 2 \text { enfermeiros em qualquer } \\
\text { freqüência. }\end{array}$ & 12,4 \\
\hline 6c. Realização do(s) procedimento(s) com 3 ou mais enfermeiros em qualquer freqüência. & 17,0 \\
\hline \multicolumn{2}{|l|}{ 7. Suporte e Cuidados aos familiares e pacientes } \\
\hline $\begin{array}{l}\text { 7a. Suporte e cuidado aos familiares e pacientes que requerem dedicação exclusiva por cerca de uma hora } \\
\text { em algum plantão. }\end{array}$ & 4,0 \\
\hline $\begin{array}{l}\text { 7b. Suporte e cuidado aos familiares e pacientes que requerem dedicação exclusiva por } 3 \text { horas ou mais em } \\
\text { algum plantão. }\end{array}$ & 32,0 \\
\hline \multicolumn{2}{|l|}{ 8. Tarefas administrativas e gerenciais } \\
\hline 8a. Realização de tarefas de rotina. & 4,2 \\
\hline $\begin{array}{l}\text { 8b. Realização de tarefas administrativas e gerenciais que requerem dedicação exclusiva por cerca de } 2 \text { horas } \\
\text { em algum plantão. }\end{array}$ & 23,2 \\
\hline $\begin{array}{l}\text { 8c. Realização de tarefas administrativas e gerenciais que requerem dedicação integral por cerca de } 4 \text { horas } \\
\text { ou mais de tempo em algum plantão. }\end{array}$ & 30,0 \\
\hline $\begin{array}{l}\text { 9. Suporte respiratório: Qualquer forma de ventilação mecânica/ventilação assistida com ou sem pressão } \\
\text { expiratória final positiva, com ou sem relaxantes musculares; respiração espontânea com ou sem pressão } \\
\text { expiratória final positiva (ex: CPAP ou BIPAP), com ou sem tubo endotraqueal; oxigênio suplementar por } \\
\text { qualquer método. }\end{array}$ & 1,4 \\
\hline 10. Cuidado com vias aéreas artificiais: Tubo endotraqueal ou cânula de traqueostomia. & 1,8 \\
\hline $\begin{array}{l}\text { 11. Tratamento para melhora da função pulmonar. Fisioterapia torácica, espirometria estimulada, terapia } \\
\text { inalatória, aspiração endotraqueal. }\end{array}$ & 4,4 \\
\hline
\end{tabular}




\begin{tabular}{|l|c|}
\hline \multicolumn{1}{|c|}{ NAS } & Pont. \\
\hline 12. Suporte cardiovascular: Medicação vasoativa independente do tipo e dose. & 1,2 \\
\hline $\begin{array}{l}\text { 13. Reposição intravenosa de grandes perdas de fluídos. Administração de fluídos }>31 / \mathrm{m}^{2} / \text { dia, qualquer tipo de } \\
\text { fluído. }\end{array}$ & 2,5 \\
\hline 14. Monitorização do átrio esquerdo. Cateter da artéria pulmonar com ou sem medida do débito cardíaco. & 1,7 \\
\hline 15. Reanimação cardiorrespiratória nas últimas 24 horas (excluído soco precordial). & 7,1 \\
\hline 16. Suporte renal: Técnicas de hemofiltração. Técnicas dialíticas. & 7,7 \\
\hline 17. Medida quantitativa do débito urinário (ex. sonda vesical de demora). & 7,0 \\
\hline 18. Suporte neurológico: Medida da pressão intracraniana. & 1,6 \\
\hline 19. Suporte metabólico: Tratamento da acidose/alcalose metabólica complicada. & 1,3 \\
\hline 20. Hiperalimentação intravenosa. & 2,8 \\
\hline 21. Alimentação enteral. Através de tubo gástrico ou outra via gastrointestinal (ex: jejunostomia). & 1,3 \\
\hline 22. Intervenções específicas: & 2,8 \\
\hline 23. Intervenções específicas fora da unidade de terapia intensiva. & 1,9 \\
\hline * Os itens 1,4,6,7 e \& são mutuamente excludentes & \\
\hline
\end{tabular}

